\title{
Data from the Romanian Registry of Rheumatic Diseases for patients with psoriatic arthritis treated with biologic disease-modifying anti-rheumatic drugs during $\mathbf{2 0 1 9}$
}

\author{
Andra Balanescu ${ }^{1,4}$, Corina Mogosan ${ }^{2,4}$, Magda Parvu ${ }^{3}$, Ruxandra Ionescu ${ }^{1,4}$ \\ ${ }^{1}$ Department of Internal Medicine and Rheumatology, "Sf. Maria" Hospital, Bucharest, Romania \\ 2"Dr. Ion Stoia" Clinical Center for Rheumatic Diseases, Bucharest, Romania \\ ${ }^{3}$ Department of Rheumatology, Colentina Hospital, Bucharest, Romania \\ 4"Carol Davila"University of Medicine and Pharmacy, Bucharest, Romania
}

\begin{abstract}
The Romanian Register of Rheumatic Diseases (RRBR, in Romanian) is an electronic database which includes data regarding all patients with immune-mediated rheumatic diseases who receive biologic therapies. These real-life data include: demographics, clinical disease forms, comorbidities, treatment regimens with biologic disease-modifying anti-rheumatic drugs (bDMARDs), and efficacy and safety data related to these treatments. The purpose of this study was to analyse the data of patients with psoriatic arthritis (PsA) introduced in the RRBR in 2019. The results showed that the number of patients whose records were included in the RRBR was relatively constant compared to previous years. Patients with PsA had numerous comorbidities, with cardiovascular being the most commonly encountered. Most patients continue to receive combination therapy with a conventional synthetic DMARD (CSDMARD), methotrexate being the most commonly used, but the percentage was lower than 2018. The concomitant use of systemic glucocorticoids has been recorded at a very low percentage. There is a tendency to initiate biologic treatment earlier, with over one third of patients receiving biologic therapy within the first 2 years after diagnosis. The most prescribed bDMARDs are still TNF blockers, with etanercept (original + biosimilar) being the most common, followed by adalimumab (original + biosimilar). The only biologic agent with another mechanism of action that was reimbursed in Romania in 2019 was secukinumab, this drug being used in almost one third of initiation and being the main option in the case of switches. The efficacy data show that the vast majority of patients have a low disease activity or are in remission. Although the percentage of patients who had a tapering regimen was reduced, this strategy proved to be a valid option for the majority of those to which it was applied. RRBR is an extremely useful tool for evaluating real-life data related to patients with PsA and the therapeutic strategies used in Romania, allowing their integration and interpretation in the context of similar registers in other countries.
\end{abstract}

Keywords: psoriatic arthritis, Romanian Registry of Rheumatic Diseases, biologics

\section{INTRODUCTION}

Psoriatic arthritis (PsA) is a chronic, immune-mediated inflammatory disease, which is part of the group of spondyloarthritis (SpA), characterized by complex axial and peripheral arthritis, skin involvement, and multiple extra-articular manifestations. Recent years have brought many changes in the therapeutic approach of PsA, with the introduction of new drugs, both biologic and targeted synthetic disease-modifying anti-rheumatic drugs (bDMARDs, tsDMARDs) into practice, even if compared to rheu- matoid arthritis (RA) their number is smaller. The heterogeneous nature of the disease, the particularities of the genetic background, the differences between medical systems, national protocols and access to therapy make real life data regarding this disease extremely valuable.

National registries are extremely important tools for providing real-life data. They represent a collection of information on a disease or group of diseases, focused on assessing demographic particularities, clinical forms, comorbidities, therapeutic schemes, 
effectiveness and safety data. Moreover, registries can collect first-hand information able to track trends about the patients' population, treatments strategies and more.

In Romania, the Romanian Register of Rheumatic Diseases (RRBR) was launched in 2013 and it includes all patients with RA, PsA, SpA, systemic lupus erythematosus and vasculitis receiving biologic treatment. RRBR is a national electronic database, which belongs to the Association "Romanian Registry of Rheumatic Diseases", a non-profit organization initiated by the Romanian Society of Rheumatology (1). The current study presents and analyzes the demographic characteristics of patients included in the RRBR in 2019, as well as treatment protocols with bDMARDs. The dynamics of different bDMARDs were followed.

\section{MATERIALS AND METHODS}

The RRBR data for PsA patients introduced in 2019 were analyzed and processed with the program.

Data are added to the RRBR by the treating rheumatologist. Entering patient data into RRBR being mandatory in order to prescribe biologic therapy to a patient, after the patient signed an informed consent for both treatment option and scientific use of their data.

The initial file includes demographic data, medical history, disease history including all previous treatments, clinical evaluation assessed using DAP$\mathrm{SA}$, the results of lab tests (including acute phase reactants), the shared decision between patient and physician regarding the bDMARD to be introduced. In order to benefit from the biologic treatment, the patient must meet the criteria required by the Romanian National Protocol (2):

- Positive diagnosis of PsA (classified according to CASPAR criteria);

- Active PsA with DAPSA $>28$, more than 5 tender/swollen joints;

- $\quad$ ESR $>28 \mathrm{~mm} / 1 \mathrm{~h}$ and CRP $>3 \mathrm{x}$ upper limit of the normal value (quantitative);

- No response to at least 2 csDMARD at doses and duration as specified in the protocol;

- No response to one csDMARD at doses and duration as specified in the protocol (for PsA with unfavourable prognostic factors);

- Two visual analog scale (VAS; global and pain), laboratory screenings, and analyses according to the RRBR Mandatory File;
- Absence of contraindications recognized for biologic therapy;

- Patient file included in RRBR.

Subsequently, data are updated every 6 months, except reporting of adverse events, which may be done at any time (1).

As such, in the RRBR there are 3 types of files, depending on the therapeutic decision:

- Initiations - PsA patients who fulfil the above criteria and will receive a bDMARD reimbursed by the National Health Insurance House and initial monitoring - PsA patients currently treated with a bDMARD which was not reimbursed in the beginning by the National Health Insurance House (for example, patients included in clinical trials, with a good clinical response at the end of the trial or patient initiated on a bDMARDs in other countries);

- Continuations - PsA patients with a good therapeutic response, who at 6 months reassessments visits will continue their previous reimbursed bDMARD;

- Switches - PsA patients with adverse events, primary or secondary non-responders who will not continue with their previous reimbursed bDMARD, but will receive another drug with the same of a different mode of action.

The aim of this observational prospective study was to provide characteristic data from the RRBR for the PsA population treated with biologics in Romania during 2019.

\section{RESULTS}

The number of visits introduced in the RRBR in 2019 was 849 , which represents an insignificant increase compared to the previous years: 832 in 2018 $(+2 \%)$ and 804 in $2017(+3.5 \%)$.

\section{Ps A patients characteristics}

Demographic data are shown in Table 1. There is an equal distribution by sex, with an average age of 55.8 years, $60.6 \%$ being in the age range $46-65$ years. The percentage of patients under 25 was small $(0.7 \%)$. The majority of patients live in the urban area $(71.2 \%)$ and have average education. The percentage of smokers is relatively low $(6.95 \%)$. 
TABLE 1. Characteristics of PSA patients from RRBR in $2019(n=849)$

\begin{tabular}{|l|c|}
\hline Sex: women (n, \%) & $430(50.5 \%)$ \\
& $\mathrm{F}: \mathrm{M}=1: 1$ \\
\hline Age (years, mean) & $55.83 \pm 11.57$ \\
& $\mathrm{~F}=56.5 ; \mathrm{M}=55.1$ \\
\hline Body Mass Index $\left(\mathrm{kg} / \mathrm{m}^{2}\right)$ & $28.48 \pm 5.11$ \\
\hline Residence: & \\
- urban (\%) & $605(71.2 \%)$ \\
\hline Education: & $5(<1 \%)$ \\
- illiterate & $150(18 \%)$ \\
- elementary & $473(55 \%)$ \\
- high school & $221(26 \%)$ \\
- university & \\
\hline Professional activity: & $370(43.5 \%)$ \\
- employed & $279(33 \%)$ \\
- PsA-retired & $200(23.5 \%)$ \\
- age-retired & $59(6.95 \%)$ \\
Smokers (\%) & \\
\hline
\end{tabular}

The presence of comorbidities is an extremely important aspect in PsA, which can be also associated with the relatively high percentage of elderly patients (Figure 1). Thus, over half of the patients had cardiovascular disease (hypertension, coronary ischemic disease, heart failure, stroke, or peripheral vascular disease), 19\% dyslipidemia, 15\% diabetes. Worth noting that in the last year there have been no new case of neoplasia.

The average duration of the disease was increased, 11.82 years. Patients included in RRBR have all a form of peripheral arthritis. In addition, $11 \%$ associated axial involvement and $20 \%$ sacroiliitis. Enthesitis was identified in $6 \%$ of patients and dactylitis in $4.6 \%$, while ocular involvement was identified in $1.5 \%$.
In 2019, there were 10 new cases of latent TB (diagnosed by positive Quantiferon test), with no new cases of hepatic viral infections.

\section{Treatment regimens of PsA patients in 2019}

The majority of patients $(692,81.5 \%)$, undergo combination therapy at the end of 2019 , which is a decrease compared to 2018 (713) (Figure 2). There are data that concomitant use of MTX would reduce immunogenicity, which is one of the reasons why many rheumatologists prefer the combination therapy $(3,4)$.

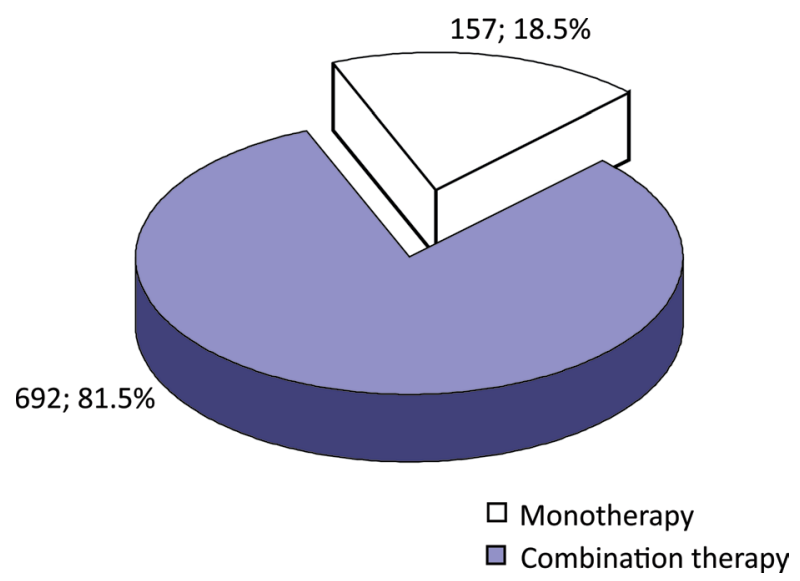

FIGURE 2. Monotherapy vs. combination therapy with csDMARDs in PsA in 2019 ( $n=849)$

It is noted that methotrexate (MTX) represents the most frequent option (65.5\%), the average dose being $15 \mathrm{mg} /$ week (most patients, 43\%, having a dose of $20 \mathrm{mg} /$ week) followed by leflunomide (LEF) $(30 \%)$ and sulfasalazine (SSZ) (14\%) (Figure 3).

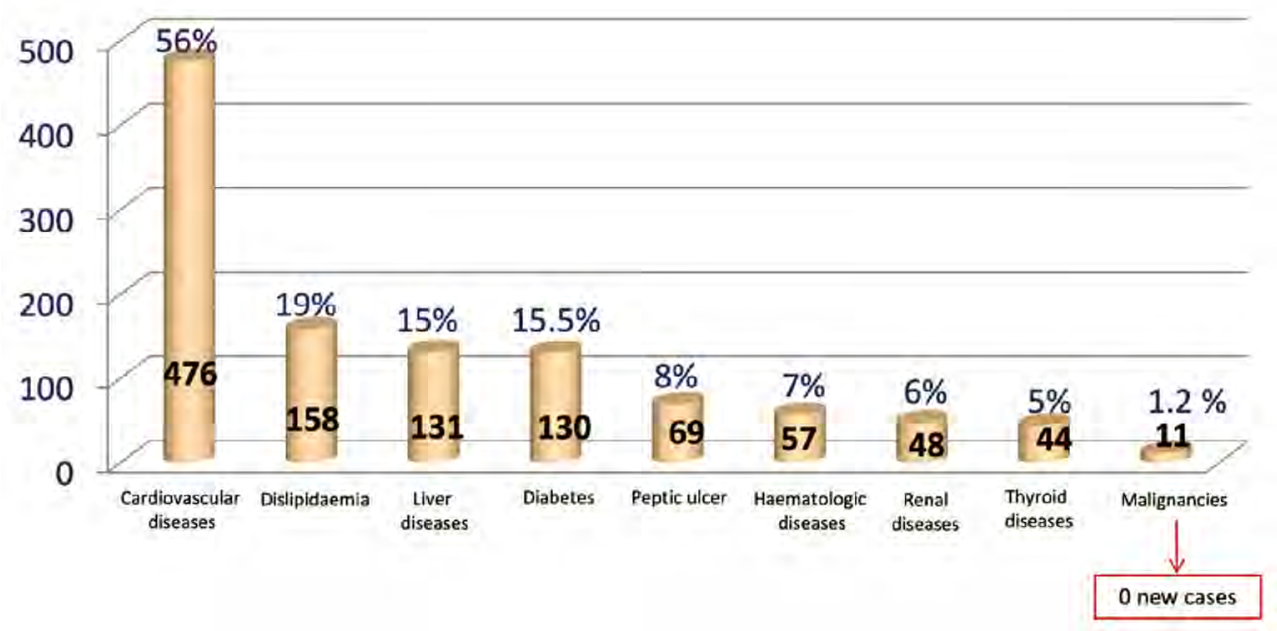

FIGURE 1. The frequency of comorbidities of PSA patients in 2019 from the RRBR. Reported values represent the percent from the total sample $(n=849)$ 


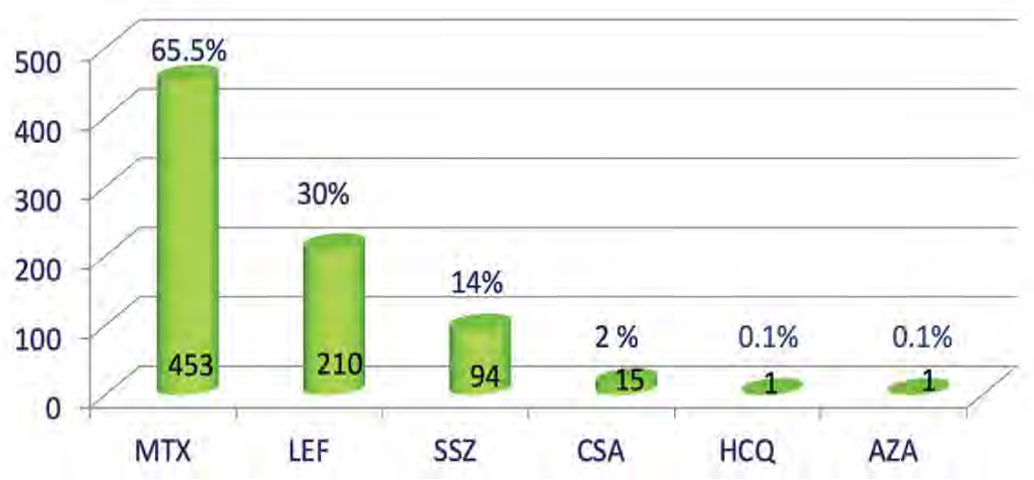

FIGURE 3. The frequency of treatment with csDMARDs of PSA patients from the $R R B R$ in 2019. Reported values represent the percent from the total sample $(n=849)$

There are still $12 \%$ of patients using a combination of more than 2 csDMARD and in these combinations, MTX is the most frequently present (MTX $+\mathrm{LEF}-5 \%$, MTX $+\mathrm{SSZ}-4.3 \%$ ) (Figure 4).

Systemic glucocorticoid therapy is rarely associated, registered in only $1.5 \%$ of patients, while local steroids were given in $5 \%$.
There is a tendency to initiate earlier biologic treatment, in $38.5 \%$ of patients receiving biologic therapy in the first 2 years, $28 \%$ between 2 and 5 years, $19 \%$ between 5 and 10 years and only $14 \%$ after 10 years (Figure 5).

The dynamics of these patients were the following: the number of continuations increased slightly

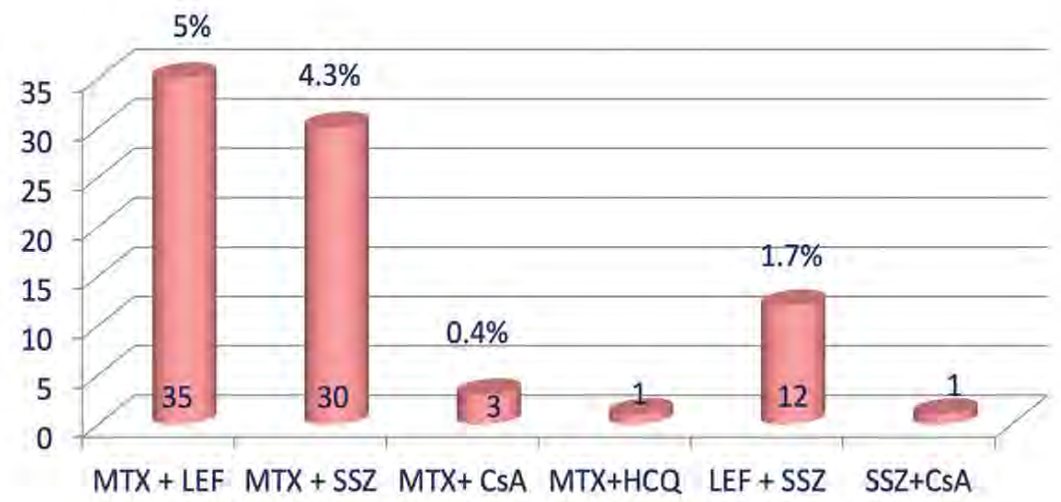

FIGURE 4. The frequency of treatment with several csDMARDs of PsA patients from the RRBR in 2019. Reported values represent the percent from the total sample $(n=849)$

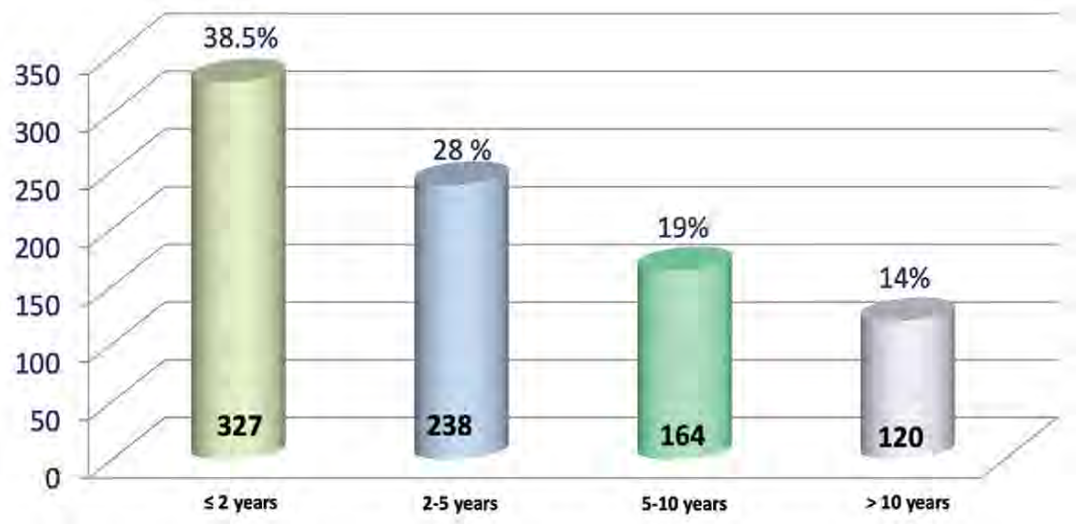

FIGURE 5. The distribution of PsA patients starting bDMARDs in 2019 according to disease duration sub-groups 


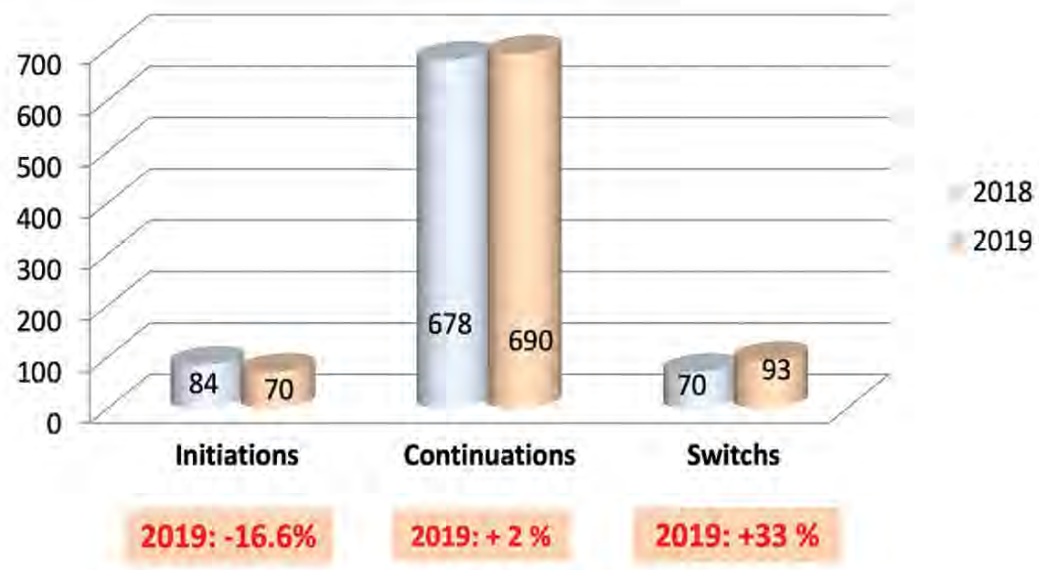

FIGURE 6. The number of PsA patients from RRBR in 2019 compared to 2018, regarding treatment decisions

$(2 \%)$, the number of initiations was lower (from 84 in 2018 to 70 in 2019), and the number of treatment changes increased from 70 in 2018 to 93 in 2019 (Figure 6).

The distribution of biologic agents used in 2019 is illustrated in Figure 7. It is observed that the most commonly used are TNF blockers, first of all being etanercept (original + biosimilar present on the Romanian market representing 38.7\%), followed by adalimumab (original + biosimilar), $31.5 \%$. The absorption of biosimilars, compared to the previous year, was higher, representing $7.8 \%$ of the total prescriptions.

The only biologic agent with another mechanism of action reimbursed in Romania in 2019 in PsA was secukinumab, the percentage of patients treated with it being $11 \%$. It should be mentioned that only in the second half of 2019, patients who were non-responders of a TNF blocker could be switched to the IL-17 inhibitor, for which the required dose is $300 \mathrm{mg} / 4$ weeks.

Regarding the biologic agents with which initiations were made, TNF blockers represented $71.55 \%$ (of which the original and biosimilar etanercept were the most used). However, an increase of the initiations on secukinumab, which represented $28.5 \%$ of the initiations has noticed (Figure 8).

Switches represents the number of those patients who will no longer be treated with a drug and will start treatment with another drug. The total number of switches in 2019 was 93 (11\%), with 11 multiple switches. The dynamic of the entries and exits of a given treatment is represented in the Figure 9 and shows a trend of change on secukinumab.

About $70 \%$ of patients follow the biologic starting product, which shows good retention. However, the remaining $30 \%$ of patients underwent treatment with 2 or more bDMARDs (Figure 10).

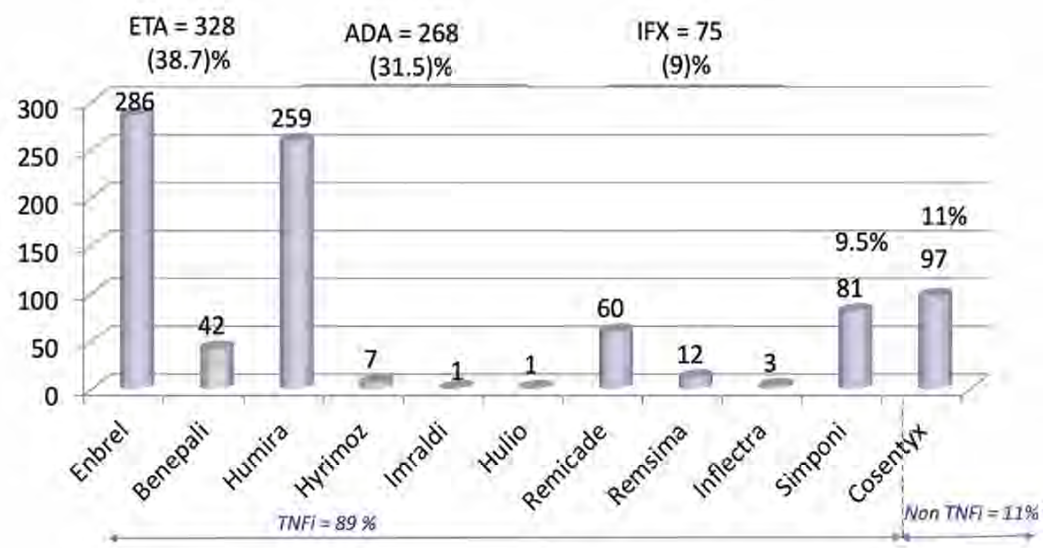

FIGURE 7. The frequency of bDMARDs (originals and biosimilars) in PSA in $2019(n=849)$ 


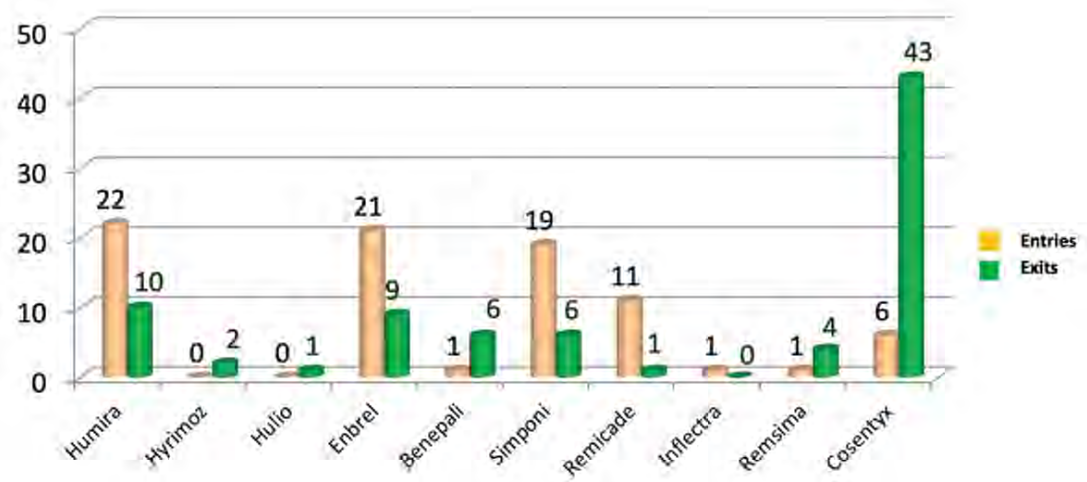

FIGURE 8. The frequency of bDMARDs (originals and biosimilars) initiations in PsA in 2019 ( $n=849)$

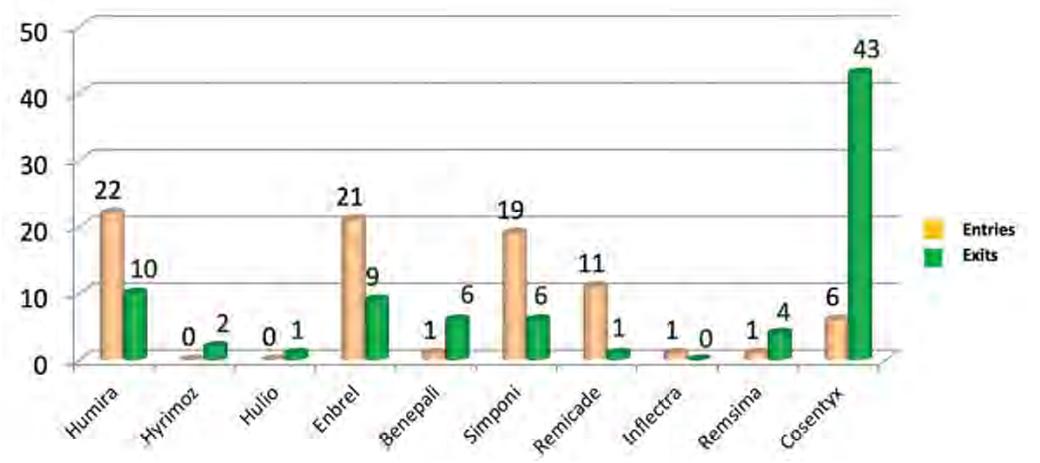

FIGURE 9. The dynamic of switches in PSA in $2019(n=849)$

\section{Ps A treatment efficacy in 2019 in the RRBR database}

The therapeutic targets in PsA should be remission or, alternatively, minimal/low disease activity $(5,6)$. The Disease Activity Index for Psoriatic Arthritis (DAPSA) is a valid and discriminative tool (7). DAPSA constitutes a disease-specific and feasible tool, especially for rheumatologists, being limited by the lack of skin evaluation. The DAPSA is simply calculated by summing swollen + tender joint counts (using 66/68 joint counts)+ patient pain
+ patient global assessments + CRP. For these reasons, the national guidelines for PsA treatment and consequently the RRBR use this composite index to assess the efficacy of the treatment in PsA.

RRBR efficacy data for patients in whom the biologic treatment was initiated, there was a significant improvement in the average value of DAPSA from 44.3 to 8.43 after 6 months (Figure 11).

The cut-off values of DAPSA are: $\leq 4$ for remission, $\leq 14$ for low disease activity, between $>14$ and $\leq 28$ for medium disease activity and $>28$ high dis-

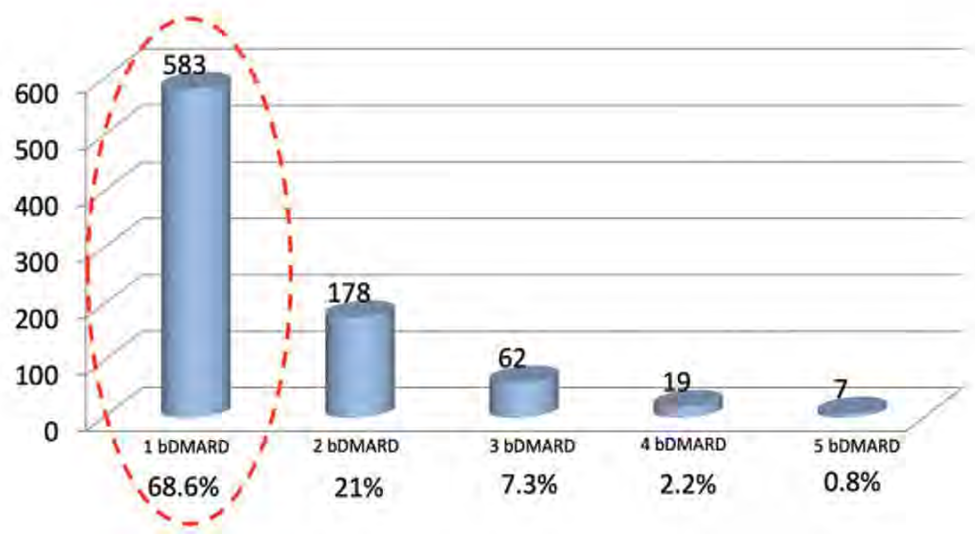

FIGURE 10. PSA patients in RRBR in 2019 according to the number of $b D M A R D$ s used 
DAPSA

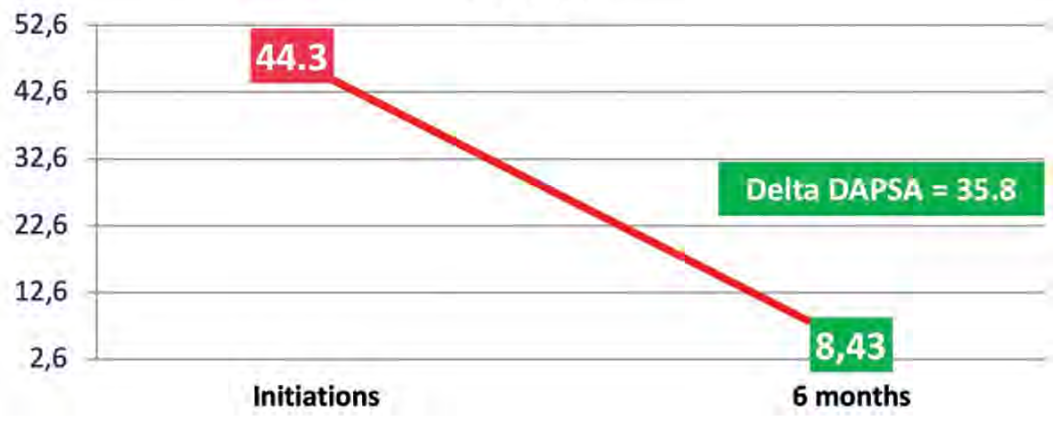

FIGURE 11. The variation of mean DAPSA after the first 6 months of treatment with $b D M A R D$ s for patients who were initiated in 2019 and had their first 6-month evaluation in 2019

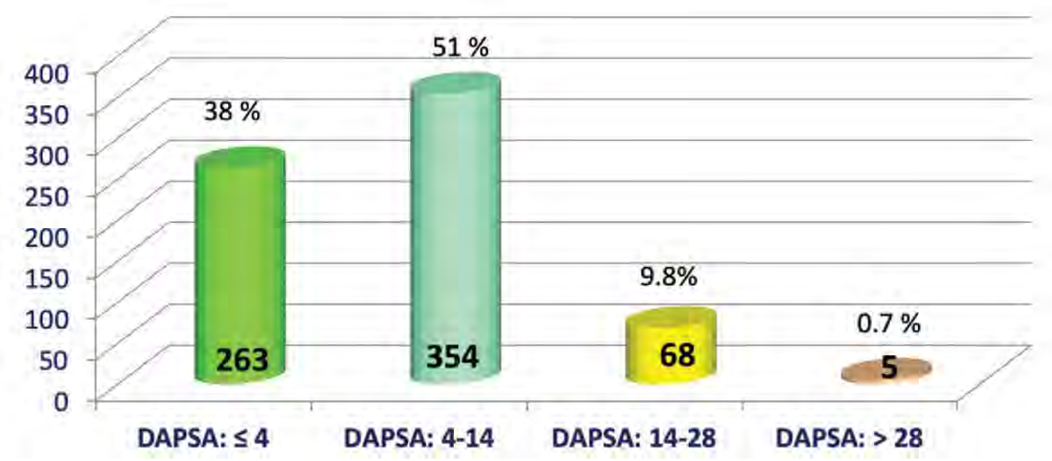

FIGURE 12. The distribution of PSA patients according to DAPSA value in 2019 in $R R B R$

ease activity (7). The distribution of PsA patients according to DAPSA value shows that half of the patients are in low disease activity and $38 \%$ in remission (Figure 12). Only 9.8\% have a medium disease activity and $0.7 \%$ have high disease activity.

The average DAPSA score is higher in those who have been treated with more biologics, which means that the switch is practiced in severe cases, with a higher disease activity, which responds more difficult to therapy.

There was also a slight increase in the number of patients who were taped (from $5.4 \%$ to $6.8 \%$ ), and this strategy worked because only 9 patients were required to resume the initial schemes.

\section{DISCUSSION}

Although randomized controlled trials (RCTs) represent the most important data sources regarding the efficacy and safety of a treatment, real-life data offer complementary information, with less constraints and without rigid inclusion and exclusion criteria. In this context, registries are an extremely valuable tool that includes real-life data and allow a more comprehensive view of the population as a whole. The registries also have some disadvantages: sometimes incomplete data, lack of control groups, less rigorous information entry. Moreover, the purposes of RCTs and registries are different. The latter have as main purpose the evaluation of demographics, clinical data, therapeutic strategies, data on the safety of therapies (8). Internationally, several registries regarding PsA have been developed, even though their number is smaller than those dedicated to other pathologies (9).

RRBR is a national registry that includes all patients with immune-mediated rheumatic diseases who undergo biologic treatment. Of these, PsA patients whose data were included in the RRBR in 2019 were analyzed in this study. This analysis provides interesting demographic characteristics, insights into the persistence and switching of bDMARDs, treatment strategies, efficacy and safety data regarding Romanian PsA patients.

The analysis of the demographic data shows that the population of PsA patients in the RRBR is similar to that of other comparable registries: an average 
age of about 55 years, with an approximately equal sex distribution (10). This average age is slightly higher than in some RCTs (11). Also, the association of comorbidities showed similar data, with some particularities. In RRBR, cardiovascular disease was found in more than half of patients, which corresponds to data from other national registries (12). In contrast, dyslipidaemia, diabetes or malignancies were found to be smaller than in other databases $(10,13,14)$.

The proportion of patients using systemic corticosteroids is very low in RRBR, while other data show that up to $33 \%$ of patients are on corticosteroids $(10,15)$.

As in many other registries, in RRBR, most patients follow a combination therapy between a csDMARD and a bDMARD, despite the fact that the scientific arguments regarding the superior efficacy of the combination are debatable $(16,17)$.

Until 3 years ago, TNF blockers were the only bDMARD reimbursed in Romania. After secukinumab reached the Romanian market, it was reimbursed only to naive TNF blockers patients. Only from the second half of 2019, the IL-17 blocker was reimbursed for all PsA patients who met the criteria of the national protocol. Moreover, other bDMARDs, such as the IL12/23 blocker ustekinumab, are not currently reimbursed. Thus, TNF blockers are the most widely used bDMARDs for PsA patients in RRBR, but in 2019 there was a tendency to

\section{REFERENCES}

1. Codreanu C, Mogosan C, lonescu R, Ancuta I, Opris D. Biologic Therapy in Rheumatoid Arthritis: Results from the Romanian Registry of Rheumatic Diseases One Year After Initiation. Farmacia 2014;62(6):1089-96

2. http://www.cnas.ro/media/pageFiles/Lista\%20protocoalelor $\% 20$ terapeutice\%20\%20-\%20iunie\%202019.pdf.

3. Zisapel M, Zisman D, Madar-Balakirski N, et al. Prevalence of TNF-alpha blocker immunogenicity in psoriatic arthritis. The Journal of Rheumatology 2015;42:73-8.

4. Behrens F, Canete JD, Olivieri I, van Kuijk AW, McHugh N, Combe B. Tumour necrosis factor inhibitor monotherapy vs combination with MTX in the treatment of PsA: A systematic review of the literature. Rheumatology (Oxford, England) 2015;54:915-26.

5. Gossec L, Smolen JS, Ramiro S, et al. European League Against Rheumatism (EULAR) recommendations for the management of psoriatic arthritis with pharmacological therapies: 2015 update. Annals of the Rheumatic Diseases 2016;75:499-510.

6. Smolen JS, Schols M, Braun J, et al. Treating axial spondyloarthritis and peripheral spondyloarthritis, especially psoriatic arthritis, to target: 2017 update of recommendations by an international task force. Annals of the Rheumatic Diseases 2018;77:3-17.

7. Schoels MM, Aletaha D, Alasti F, Smolen JS. Disease activity in psoriatic arthritis (PsA): defining remission and treatment success increase prescriptions for secukinumab, especially at initiation and switches, trend observed in other studies as well $(10,18,19)$.

The efficacy data included in RRBR show that the therapeutic targets recommended by EULAR were reached in a significant percentage of patients, as shown by the information from both RCTs and registries.

The present study is limited by the fact that the analyzed data refer only to the year 2019, thus being unable to provide information regarding the persistence to the treatment and did not intend to analyze the safety data.

\section{CONCLUSIONS}

Information regarding baseline demographic, disease characteristics, medical history, medication regimens, therapeutic strategies, how well the disease is controlled, are extremely useful in clinical practice, because results of this real-world studies can help treatment decisions when selecting patients with PsA for biologic therapies. Treatment patterns in PsA have changed in recent years, so this kind of data from a given country or geographic area are important to inform treatment decisions and identify unmet needs. The RRBR had similarities with previously published registries, supporting its external validity, but more comprehensive analyses are needed.

Conflict of interest: none declared Financial support: none declared

using the DAPSA score. Annals of the Rheumatic Diseases 2016;75:811-8.

8. Sarzi-Puttini P, Varisco V, Ditto MC, Benucci M, Atzeni F. Psoriatic Arthritis Registries. The Journal of Rheumatology, Supplement 2015;93:30-2.

9. Kremer JM. The Corrona US registry of rheumatic and autoimmune diseases. Clinical and Experimental Rheumatology 2016;34:S96-s9.

10. Schneeweiss M, Merola JF, Karlson EW, Solomon DH. Rationale and Design of the Brigham Cohort for psoriasis and psoriatic arthritis registry (COPPAR). BMC Dermatology 2017;17:11.

11. Strober $B$, Karki $C$, Mason $M$, et al. Characterization of disease burden, comorbidities, and treatment use in a large, US-based cohort: Results from the Corrona Psoriasis Registry. Journal of the American Academy of Dermatology 2018;78:323-32.

12. Kavanaugh A, Papp K, Gottlieb AB, et al. Demography, baseline disease characteristics, and treatment history of psoriasis patients with self-reported psoriatic arthritis enrolled in the PSOLAR registry. BMC Rheumatology 2018;2:29.

13. Mease PJ, Karki C, Palmer JB, et al. Clinical Characteristics, Disease Activity, and Patient-Reported Outcomes in Psoriatic Arthritis Patients With Dactylitis or Enthesitis: Results From the Corrona Psoriatic Arthritis/Spondyloarthritis Registry. Arthritis Care \& Research 2017;69:1692-9. 
14. Lauper K, Courvoisier DS, Chevallier P, Finckh A, Gabay C. Incidence and Prevalence of Major Adverse Cardiovascular Events in Rheumatoid Arthritis, Psoriatic Arthritis, and Axial Spondyloarthritis. Arthritis Care \& Research 2018;70:1756-63.

15. Mease PJ, Karki C, Liu M, et al. Baseline patient characteristics associated with response to biologic therapy in patients with psoriatic arthritis enrolled in the Corrona Psoriatic Arthritis/ Spondyloarthritis Registry. RMD Open 2018;4:e000638.

16. Mease PJ, Lesperance T, Liu M, et al. Changes in Treatment Patterns in Patients with Psoriatic Arthritis Initiating Biologic and Nonbiologic Therapy in a Clinical Registry. The Journal of Rheumatology 2017:44:184-92.
17. Kalyoncu U, Bayindir O, Ferhat Oksuz M, et al. The Psoriatic Arthritis Registry of Turkey: results of a multicentre registry on 1081 patients. Rheumatology (Oxford, England) 2017;56:279-86.

18. Ritchlin CT, Stahle M, Poulin Y, et al. Serious infections in patients with self-reported psoriatic arthritis from the Psoriasis Longitudinal Assessment and Registry (PSOLAR) treated with biologics. BMC Rheumatology 2019;3:52.

19. Mease PJ, Karki C, Liu M, et al. Discontinuation and switching patterns of tumour necrosis factor inhibitors (TNFis) in TNFinaive and TNFi-experienced patients with psoriatic arthritis: An observational study from the US-based Corrona registry. RMD Open 2019;5:e00080. 